\title{
HISTOPATHOLOGICAL EFFECT OF SPIRULINA IN RENAL ISCHEMIA REPERFUSION IN RATS
}

\author{
Dina Abd-Allah*, Azza Abdel-Aziz *, Amira El-Hawary, *, Fatma El-Husseini, *, Soheir Sirag * \\ Address: *Department of Pathology, Faculty of Medicine, Mansoura University, Mansoura, Egypt.
}

\begin{abstract}
Background/Aim: Ischemia/reperfusion (I/R) injury occurs in renal transplantation, and it could be a risk factor for late renal allograft failure. Several exogenous antioxidants used in prevention of renal I/R injury. Multiple experimental studies demonstrated the antioxidant effect of Spirulina. This study focused on the effects of Spirulina on histopathology of kidney subjected to I/R injury.

Methods: Renal I/R was induced in male Sprague-Dwaley rats by occluding the left renal artery then right nephrectomty. Group I was sham operated rats; group II, control (non treated rats); and group III, Spirulinatreated rats $(1000 \mathrm{mg} / \mathrm{kg} \mathrm{BW}$, orally. 7 days before I/R). Rats were sacrificed $1 \& 3$ days post reperfusion. The effects of Spirulina on both necrosis and regeneration of kidney tubules caused by renal I/R injury were assayed and scored.

Results: Spirulina pretreatment decrease the necrotic injury and improve tubular regeneration after 1 and 3days of renal I/R injury.

Conclusion: Spirulina decreased the necrotic activity index in rat kidney after I/R injury and help in tubular regeneration.
\end{abstract}

Keywords: Spirulina; ischemia reperfusion (I/R) injury; acute renal failure; outer strip outer medulla (OSOM); necrosis, tubular regeneration.

\section{INTRODUCTION}

$\mathbf{R}$ enal I/R injury clinically may occur due to systemic hypoperfusion (e.g. acute myocardial infarction) with subsequent resuscitation \& occasionally discontuation of renal blood supply e.g. in kidney transplantation (1), partial nephrectomy, renal vascular surgical operation, enucleation of renal cell carcinoma and aortic cross clamping (suprarenal or juxtra-renal aortic cross clamping for abdominal aorta) (2).

Renal I/R injury is considered one of the commonest reasons of acute renal failure (ARF) which results in increase morbidity, prolonged hospitalization and increase mortality (3).

Ischemia results in injury to and death of renal cells due to stoppage of blood flow and deoxygenation. Although reperfusion is important to renal tissue survival, it results in additional damage that leads to the renal dysfunction (4).

As a result of ischemic injury, there are regional alterations in blood flow patterns. There is sever congestion and hypo perfusion of the outer medulla that remains even though blood flow improves during reperfusion after an ischemic insult resulting in acute tubular necrosis, which plays a vital role in the pathogenesis of ARF (5).
Histological changes are aggravated by increasing ischemia time and afterward by reperfusion. True extension of ischemic injury is seen at 24 to 72 hours after reperfusion (6).

Apoptosis and necrosis have been known as mechanism of cell death in renal I/R injury however the degree of apoptosis is underestimated as it is rapidly occurring distributed process that is difficult to identify and quantify in tissues (7).

Renal tubular epithelial cells have the ability to regenerate after an ischemic injury. Minimally injured tubular cells are repaired when blood flow is re-established, whereas severely injured cells undergo necrosis or apoptosis. Morphologically, repair is characterized by the appearance of dedifferentiated epithelial cells and multiple mitotic figures. The origin of these proliferating cells still unknown, but they mostly represent surviving tubular cells that have dedifferentiated (8).

Spirulina is considered of a microscopic blue-green alga that is considers one of the natural food sources of vitamin B12 and also extremely rich in proteins, minerals, essential amino acids and fatty acids (9).

Many experimental studies suggest multiple therapeutic effects of Spirulina from decrease of cholesterol level to increase efficacy of the immune system and reduction 
of nephrotoxicity by heavy metals and drugs and radiation protection (10).

Thus, this study was performed to evaluate the histopathological effects of Spirulina on renal $\mathrm{I} / \mathrm{R}$ injury in rats.

\section{MATERIAL \& METHODS}

Materials: - Fine dark blue green powder of Spirulina Arthrospira Platensis (Sp) thankfully was provided by doctor Yassin N. Elayouty professor of phycology, Botany Department Faculty of Science, Zagazig University

Experimental Animals: - This study was conducted on thirty six male Sprague-Dwaley rats, weighing 200-250 gm, aging 3-4 months, bred and housed in the animal house of medical experimental research center, faculty of medicine, Mansoura University. The main advantages of usage of Sprague-Dwaley rats in experimental studies are their calmness and ease of handling.

\section{Experimental Groups and Surgical Procedure of Ischemia/Reperfusion:-}

The following experimental group $(\mathrm{n}=12$ rats per group) were studied

1. Group: (sham): These animals were exposed to right nephrectomy, without left renal ischemia.

2. Group: (control): These animals were exposed to left renal ischemia for 45 minutes and right nephrectomy.

3. Group: (Spirulina control group): Like control group, but animals received Spirulina $1000 \mathrm{mg} / \mathrm{kg} \mathrm{BW}$ in $2.5 \mathrm{ml}$ distilled water. Spirulina was administrated orally 7 days before ischemia.

The rats were obtained from the cage and injected with sodium thiopental at a dose of $12 \mathrm{mg} / 100 \mathrm{gm}$ BW intraperitoneally for anaethesia . After anesthesia, the animal was fixed in supine position on the surgical operating table and the skin of the abdomen was shaved and sterilized with ethyl alcohol. Then, the abdomen was entered via midline laparotomy and the left renal vascular pedicle was isolated and clamping of the left renal artery was done for 45 minutes using a Bulldog clamp. Then approximation of the edges of the abdominal incision was done and covered by gauze rich in warm isotonic saline $\left(37^{\circ} \mathrm{C}\right)$ to maintain temperature and decrease loss of fluids. Five minutes before removal of the vascular clamp, the right kidney was exposed and dissected. After that, removal of the vascular clamp on the left renal artery was done, the abdomen was irrigated by saline then the closure of abdominal incision was done.

The body temperature was maintained at $37 \pm$ $1^{\circ} \mathrm{C}$ by overhead lamp and placing gauze rich in warm saline over the abdominal incision. Rats were undergoing sacrifaction after 1 and 3 days of reperfusion. Kidney tissues were taken for analysis.

Renal Morphology: - After sacrifice the rat, opening of the abdomen was done and the left kidney was perfused with saline via a cannula put in the abdominal aorta to remove the blood. Then perfusion with formalin via the same cannula and removal of kidney and place it in container filled with $10 \%$ neutral buffered formalin for histopathological assessment. Then, tissues were dissected into $5 \mathrm{~mm}$ thin sections, deparafinized, dehydrated, and stained with H\&E. Sections prepared from rats kidney were examined by a light microscope for histopathologic evaluation in a blinded fashion.

The kidney sections were examined in each layer for necrotic activity \& regenerating activity. Necrotic activity assessed by necrotic tubules: They will be scored as number of necrotic tubules / HPF, 1-5 necrotic tubules / HPF (score 1); 5-10 necrotic tubules / HPF (2); 10-15 necrotic tubules / HPF (3) and more than 15 necrotic tubules / HPF (4). Regenerating activity assessed by five parameters which are Solid sheets of cells in the interstitium they will be counted as number / HPF, 1-2 solid sheets / HPF (1); 3-5 solid sheets / HPF (2) and more than 5 solid sheets / HPF (3). Intraepithelial tubular proliferation they will be counted as number / HPF. 1-2 solid tubules / HPF (1); 3-5 solid tubules / HPF (2) and more than 5 solid tubules HPF (3). Mitotic figures they are counted as Number /10HPFs; 1-2 /10 HPF (1); 3-5 /10 HPF (2) and more than $5 / 10$ HPFs (3). Tubules with large vesicular nuclei: Absence (0); Presence (1). Dilated tubules with festooned nuclei; Absence (0); Presence (1). So, Activity necrotic tubule scoring index was 4 and regenerating tubules scoring index was 11.

Statistical methods

Data were analyzed using the computer program SPSS version 17.0 to obtain. 
Descriptive statistics were illustrated in the form of frequency (Number-percent) and median (range). In the statistical comparison between Sham, control and spirulina groups, the significance of difference was tested using Kruskal Wallis test followed by mann-whitney for multiple comparisons. $P$ value was considered significant when it was $<0.05$ in all analyses.

\section{RESULTS}

The histopathologic parameters were assesed and illustrated in (Tables 1-4). The Sham group has no morphologic changes either necrotic or regenerative changes on various days after $\mathrm{I} / \mathrm{R}$ injury (photomicrograph 1). In contrast, the section of kidneys obtained from control group demonstrated the recognized features of significant necrotic changes (photomicrograph 2) and non-significant regenerative changes on various days of $I / R$ injury.

Spirulina treated group revealed significant decrease in OSOM necrotic activity and significant increase in OSOM \& inner medulla regenerative activity on various days of $\mathrm{I} / \mathrm{R}$ injury. These regenerative activities include solid sheet, intratubular proliferation, mitotic figures, dilated tubules lined by festooned nuclei and tubules with vesicular nuclei (figure 1)

Table (1): Necrotic activity index in all layers of the kidneys in rats sacrified one day after renal ischemia-reperfusion (median (range)).

\begin{tabular}{cccc}
\hline Activity index & Sham & Control & Spirulina + control \\
\hline Cortex & $0(0-0)$ & $1(1-2)^{\mathbf{a}}$ & $0(0-2)$ \\
\hline OSOM & $0(0-0)$ & $4(3-4)^{\mathbf{a}}$ & $2(1-3)^{\mathbf{a b}}$ \\
\hline ISOM & $0(0-0)$ & $2(1-2)^{\mathbf{a}}$ & $0(0-0)^{\mathbf{b}}$ \\
\hline Inner medulla & $0(0-0)$ & $0(0-0)$ & $0(0-0)$ \\
\hline
\end{tabular}

\section{Data illustrated as median (range)}

\section{P: Probability Test used: Kruskalwallis test followed by mann-whitney for multiple} comparisons

$a: p<0.05$ comparison with the rats of sham group

$b: p<0.05$ comparison with the rats of control group

Table (2): Regenerative index in all layers of the kidneys in rats sacrified one day after renal ischemia-reperfusion (median (range))

\begin{tabular}{llll}
\hline Regeneration index & Sham & Control & Spirulina + control
\end{tabular}

\begin{tabular}{cccc}
\hline Cortex & $0(0-0)$ & $0(0-0)$ & $0(0-0)$ \\
\hline OSOM & $0(0-0)$ & $0(0-1)$ & $1(0-1)^{\mathbf{a}}$ \\
\hline ISOM & $0(0-0)$ & $0(0-0)$ & $0(0-0)$ \\
\hline Inner medulla & $0(0-0)$ & $0(0-1)$ & $3(2-3)^{\mathbf{a b}}$
\end{tabular}

\section{Data illustrated as median (range).}

\section{P: Probability \\ Test used: Kruskalwallis test followed by mann-whitney for multiple} comparisons

$a: p<0.05$ comparison with the rats of sham group

$b: p<0.05$ comparison with the rats of control group 
Table (3): Necrotic activity index in all layers of the kidneys in rats sacrified three days after renal ischemia-reperfusion (median (range)).

\begin{tabular}{cccc}
\hline Activity index & Sham & Control & Spirulina + control \\
\hline Cortex & $0(0-0)$ & $1(0-1)^{\mathbf{a}}$ & $0(0-1)$ \\
\hline OSOM & $0(0-0)$ & $3(3-4)^{\mathbf{a}}$ & $1(0-2)^{\mathbf{b}}$ \\
\hline ISOM & $0(0-0)$ & $0(0-1)$ & $0(0-0)$ \\
\hline Inner medulla & $0(0-0)$ & $0(0-0)$ & $0(0-0)$
\end{tabular}

\section{Data illustrated as median (range).}

P: Probability Test used: Kruskalwallis test followed by mann-whitney for multiple comparisons

$a: p<0.05$ comparison with the rats of sham group

$b: p<0.05$ comparison with the rats of control group

Table (4): Regenerative index in all layers of the kidneys in rats sacrified three days after renal ischemia-reperfusion (median)

\begin{tabular}{cccc}
\hline Regeneration index & Sham & Control & Spirulina + control \\
\hline Cortex & $0(0-0)$ & $0(0-0)$ & $1(0-2)^{\text {ab }}$ \\
\hline OSOM & $0(0-0)$ & $1(0-2)$ & $7(6-8)^{\text {ab }}$ \\
\hline ISOM & $0(0-0)$ & $0(0-0)$ & $0(0-0)$ \\
\hline Inner medulla & $0(0-0)$ & $1(0-2)$ & $4(2-4)^{\text {ab }}$ \\
\hline
\end{tabular}

Data illustrated as median (range).

P: Probability Test used: Kruskalwallis test followed by mann-whitney for multiple comparisons

a: $p<0.05$ comparison with the rats of sham group

$b: p<0.05$ comparison with the rats of control group 

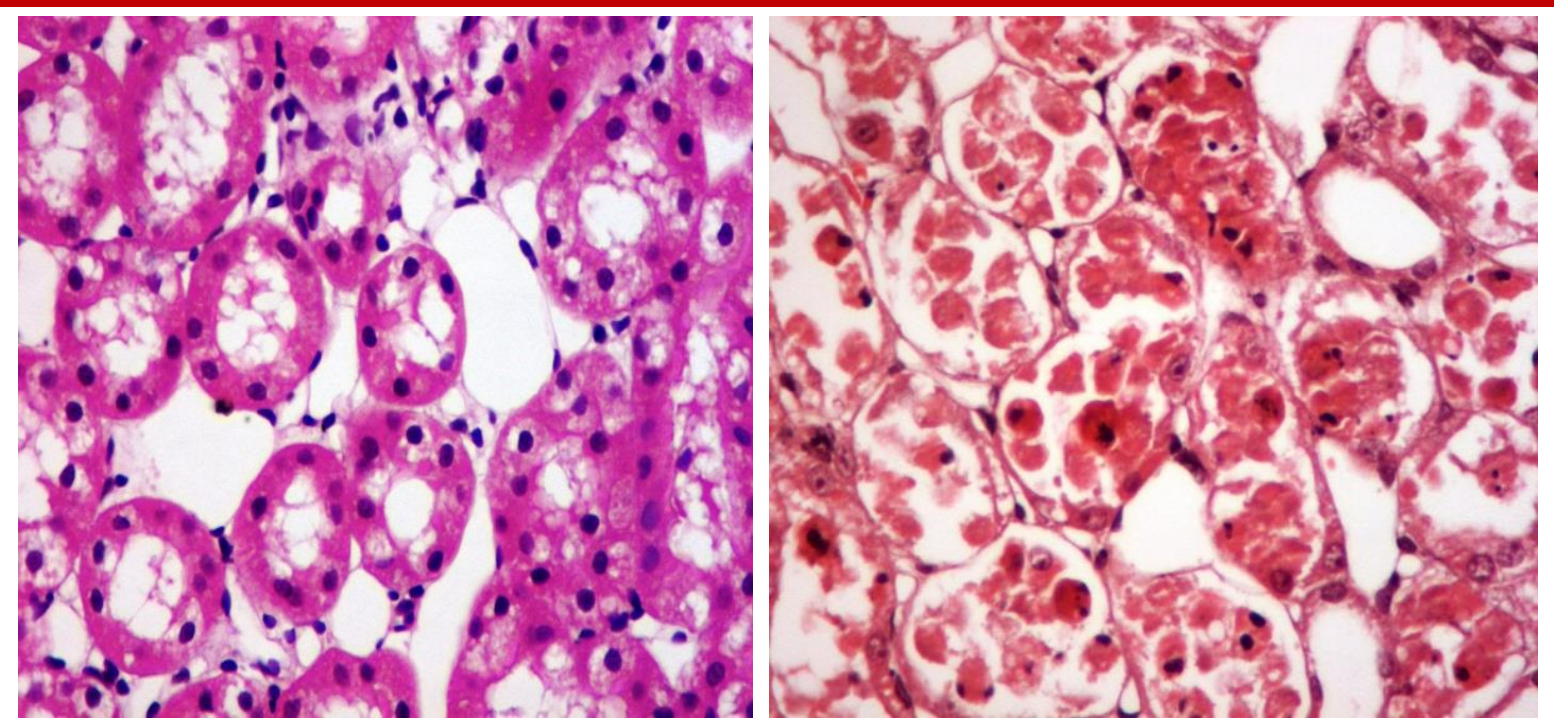

Photomicrograph (1): Sham group after 1 day. Tubules of OSOM with intact architecture and no necrotic changes (H\&E 400)
Photomicrograph (2): control group after 1 day. Tubules of OSOM show 17 necrotic tubules/ HPF score (4) (H\&E X400)
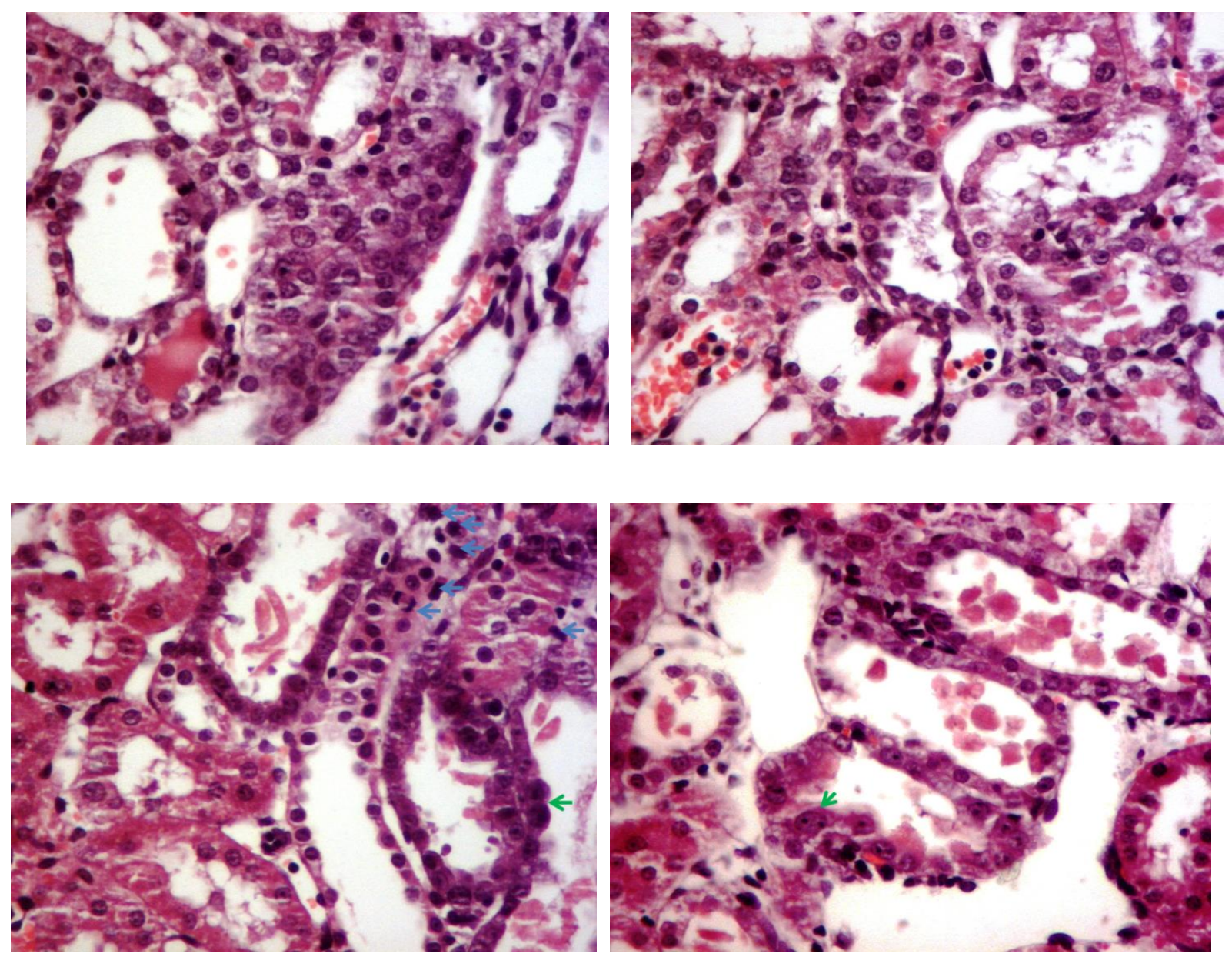

Figure 1. Different patterns of OSOM tubular regeneration; H\&E: $\times 400$. (A) Photomicrograph of 1 solid sheet. (B) Photomicrograph of 1 tubule with intratubular proliferation. (C) Photomicrograph of dilated tubule with festooned nuclei (green arrow) and 6 mitotic figures (blue arrows). (D) Photomicrograph of tubule lined by vesicular nuclei (green arrow). 


\section{DISCUSSION}

The ARF induced by $\mathrm{I} / \mathrm{R}$ injury characterized by renal dysfunction, sever tubular damage and necrosis (3). Histological analysis of renal biopsy samples still the most accurate diagnostic test for 2 major problems affecting graft survival: rejection and acute renal failure (6).

Spirulina has high nutritional benefit and with wide uses in medicinal applications. It is riches in antioxidant agents and free radical scavenging substances (11).

Some experiments showed that various exogenous antioxidant agents such as Spirulina have renoprotective antioxidant effect as the study of Karadeniz et al., (12) which study the effect of Spirulina in protection against Gentamicin-induced Nephrotoxicity in Rats and revealed improvement in histopathological parameters of kidney in the group treated with Spirulina. And also, Mohan et al., (13) study which used Spirulina on protection against cisplatininduced nephrotoxicity by Spirulina in rats.

In another study by Sinanoglu et al., (14), evaluated the role of Spirulina in the protection of cyclophosphamide (CP)-induced nephrotoxicity and urotoxicity in rats who illustrated that there were structural changes in renal tissue of cyclophosphamide treated animals and its reversal by Spirulina treated animals.

To the best of our knowledge, we think that Spirulina treatment has not been used in renal I/R injury studies. For this reason, we selected to study the histopathological effect of Spirulina on renal I/R injury. In the present study, as regard necrotic activity we observed, After 1 day of I/R injury there was significant decrease in necrotic activity in Spirulina+ control group than control group in OSOM \& ISOM. After 3 days of renal I/R injury there is significant decrease in OSOM activity in Spirulina+control group than control group.

This came in accordance with the study conducted by (Thaakur and Sravanthi) (15) study which used Spirulina as neuroprotective in cerebral $I / R$ injury and shows decrease necrotic degeneration of neuronal cells compared with ischemic control. Khan et al., (16) use C-phycolcyanin, an antioxidant biliprotein pigment of Spirulina platensis in protection of heart against $\mathrm{I} / \mathrm{R}$ injury and revealed improvement in histopathological parameters in treated group with Cphycocyanin.

Previous studies did not spot light upon reparative effect of Spirulina, however in our study, reparative effect of Spirulina was tested via assessment of tubular regeneration. After 1 day, there was significant increase difference in inner medulla regenerative activity than control group. After 3 days, Regeneration activity after 3 days of renal $\mathrm{I} / \mathrm{R}$ injury revealed significant increase in cortical, OSOM \&inner medulla than control group.

In conclusion, the findings of this study showed, that Spirulina was used to decrease tubular necrosis and also, help in tubular regeneration associated with renal I/R injury in male rats. We suggest that application of Spirulina could be helpful in clinical practice, particularly in transplantation and renal surgery. Still extensive research is required for understanding of the mechanism of action of Spirulina for providing protection and repair in cases of renal I/R injury.

\section{List of abbreviations}

I/R, ischemia reperfusion; OSOM, outer strip outer medulla; ISOM, inner strip outer medulla; ROS, reactive oxygen species; ARF, acute renal failure; HPF, high power field; $\mathrm{H} \& \mathrm{E}$, haematoxylin and eosin.

\section{REFERENCES}

1. Weight SC, Walter JR, Bradley V et al., (2001): interaction of eicosanoids \& nitric oxide in renal reperfusion injury. Tranplantatiion, 72:614-619

2. Saito M and Miyagawa I (2000): renal time monitoring of nitric oxide in ischemia reperfusion injury in rat kidney. Urol. Res, 28:141-146

3. Korkmaz A and Kolankaya D (2010): protective effect of Rutin on ischemia/ reperfusion induced damage in rat kidney. Journal of surgical research, 164: 309-315

4. Simmons M, Schreiber M and Gill I (2008): Surgical renal ischemia: a contemporary overview. J Urol, 180:19

5. Just A (2007): Mechanisms of renal blood flow autoregulation: dynamics and contributions. Am J Physiol Regul Integr Comp Physiol, 292:1-17

6. Tirapelli L, Barione D, Trazzi B et al., (2009): Comparison of two models for 
evaluation histopathology of experimental renal ischemia. Transplantation Proceedings, 41, 4083-4087

7. Joseph V and Li Yang (2011): Cellular pathophysiology of ischemic acute kidney injury. The Journal of Clinical Investigation, 121:4210-4221

8. Humphreys B, Valerius M, Kobayashi A et al., (2008): Intrinsic epithelial cells repair the kidney after injury. Cell Stem Cell,2:284-291

9. Rodríguez-Sánchez R, Ortiz-Butrón R, Blas-Valdivia V et al., (2012): Phycobiliproteins or C-phycocyanin of Arthrospira (Spirulina) maximaprotect against $\mathrm{HgCl} 2$-caused oxidative stress and renal damage. Food chemistry, 135:23592365

10. Dartsch P (2008): Antioxidant Potential of Selected Spirulina platensisPreparations. Phytother. Res., 22:627-633

11. Kuhad A, Tirkey N, Pilkhwal S et al., (2006): Effect of Spirulina, a blue green algae, on gentamicin-induced oxidative stress and renal dysfunction in rats. Fundam Clin Pharmacol, 20:121-129.
12. Karadeniz A, Yildirim A, Simsek $\mathrm{N}$ et al., (2008): Spirulina platensis Protects against Gentamicin-induced Nephrotoxicity in Rats. Phytother. Res. 22: 1506-1510

13. Mohan IK, Khan M, Shobha JC et al., (2006): Protection against cisplatin-induced nephrotoxicity by Spirulina in rats. Cancer Chemother Pharmacol, 58:802-810

14. Sinanoglu $\mathrm{O}^{1}$, Yener AN, Ekici $\mathrm{S}$ et al., (2012): The protective effects of spirulina in cyclophosphamide induced nephrotoxicity and urotoxicity in rats. Urology, 80:13921398

15. Thaakur S and Sravanthi R (2010): Neuroprotective effect of Spirulina in cerebralischemia-reperfusion injury in rats. J Neural Transm, 117:1083-1091

16. Khan M, Varadharaj S, Ganesan LP et al., (2006): C-phycocyanin protects against ischemia-reperfusion injury of heart through involvement of p38 MAPK and ERK signaling. Am J Physiol Heart Circ Physiol. 290:2136-2145. 